\title{
Eye movements and mental attentional capacity in children and adults
}

Valentina Bachurina(1); Marie Arsalidou PhD (2)

1 - National Research University Higher School of Economics, Moscow, Russia

2 - National Research University Higher School of Economics, Moscow, Russia; York University, Toronto, Canada

\section{Introduction}

- Eye-tracking is a non-invasive method that has been repeatedly used to study attention and related cognitive processes.

- While eye-tracking is not a direct measure of brain activity, it has been shown to reveal information about mental processes ${ }^{1}$.

- Mental attentional capacity (MAC) corresponds to the amount of information an individual can maintain and manipulate in mind $^{2}$

- MAC is considered the central maturational component of working memory ${ }^{3}$ and it is closely related to other aspects of cognitive competence and intelligence.

\section{The purpose of this study is to} investigate the relation between eye-tracking indices (e.g., number and duration of fixations) and MAC.

\section{References :}

Karatekin, C. (2007). Eye tracking studies of normative and atypical development. Developmental Review, 27(3), 283-348. https.//doi.org/10.1016/1.dr.2007.06.006 transition rule in Piaget's developmental stages sychologica, 32, 301-345

psychologica, 32, 301-345 \& \& Johnson, (2010). Misleading cues improve devel, \& John Jon, J. (2010). working memory capacity: the color matching tasks. Cognitive Development, 25(3), 262-277.
Method

- EyeLinkPortableDuo (SR Research portable eye-tracker)

- 33 adults ( $M=22.8 \pm 4.1,17$ male) and 15 children (age 8-9 years, 4 male)

- Colour Matching Task (CMT) visual-spatial measure of MAC (Figure 1)

- Control task - anti/pro-saccade task (Figure 2)

\begin{tabular}{cc} 
Interference & $\begin{array}{c}\text { Colour matching tasks (CMT) } \\
\text { Visuospatial }\end{array}$ \\
\hline
\end{tabular}

Low CMT-Simple

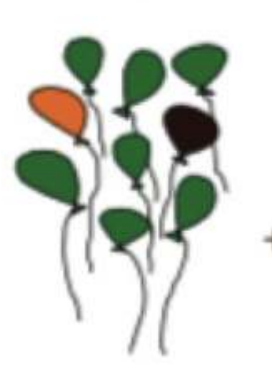

High

Figure 1. Example of CMT stimuli.

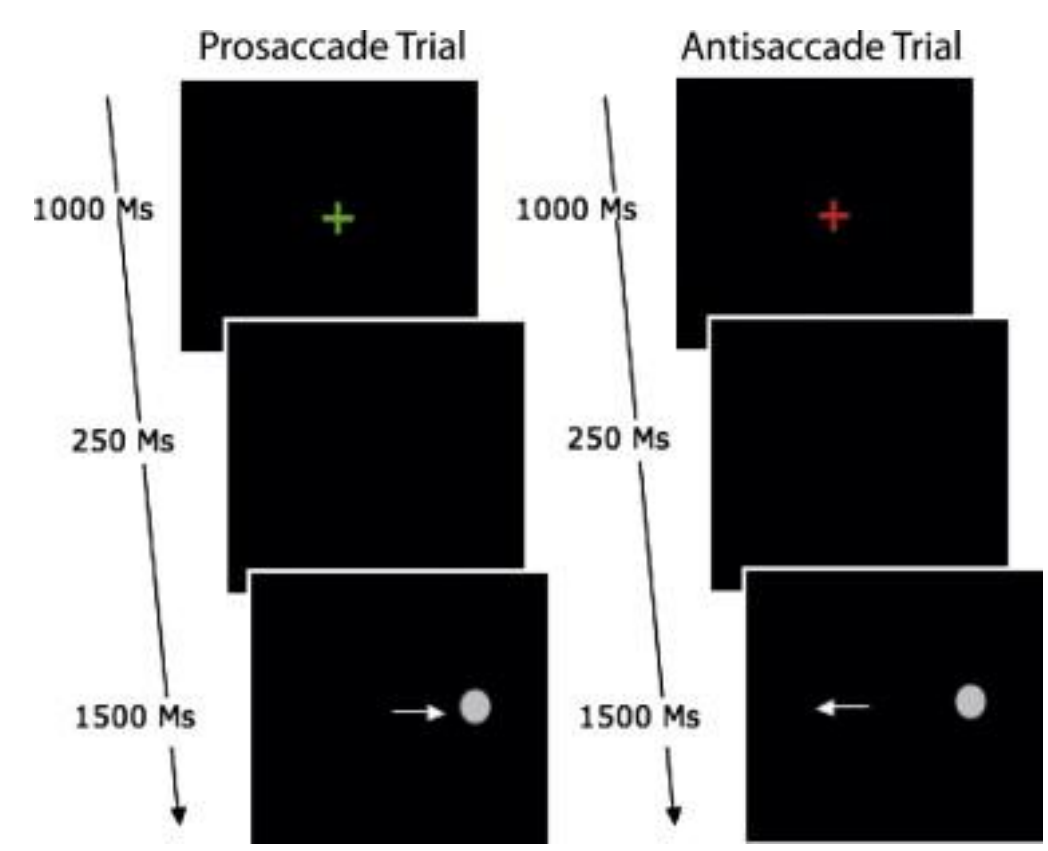

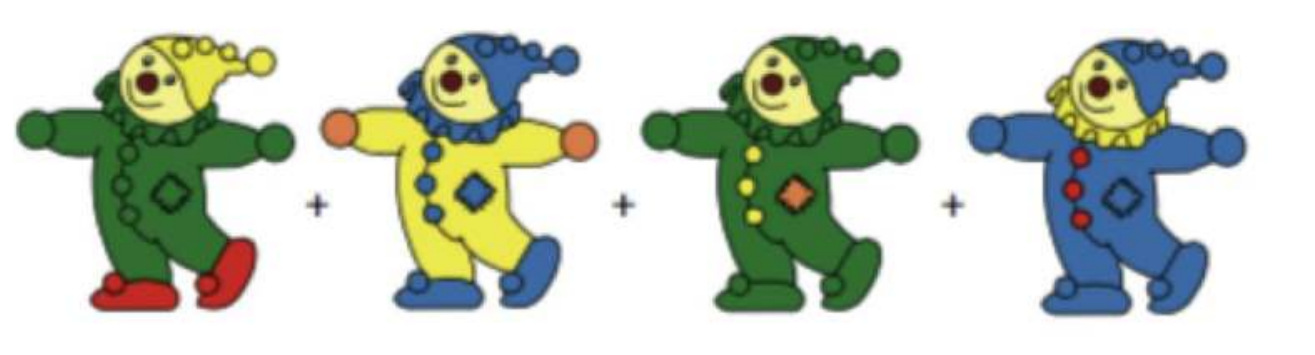

Results

- Two-way ANOVA revealed significant effect $(p<0.005)$ of both MA load and interference condition on reaction times and accuracy in the Colour matching task

- Two-way ANOVA revealed a significant effect of difficulty for number of fixations for both age groups (adults: $F=$ $50.05, p=0.000286, \eta^{2}=$ 0.96744564 and children $F$ $=5, p=6.39 \mathrm{e}-05, \mathrm{n}^{2}=$ $0.1409805)$.

- ANOVA conducted to test for effect of age group found a significant effect $(p=$ 0.00412)

- ANOVA revealed significant difference between age groups for the anti/pro-saccade

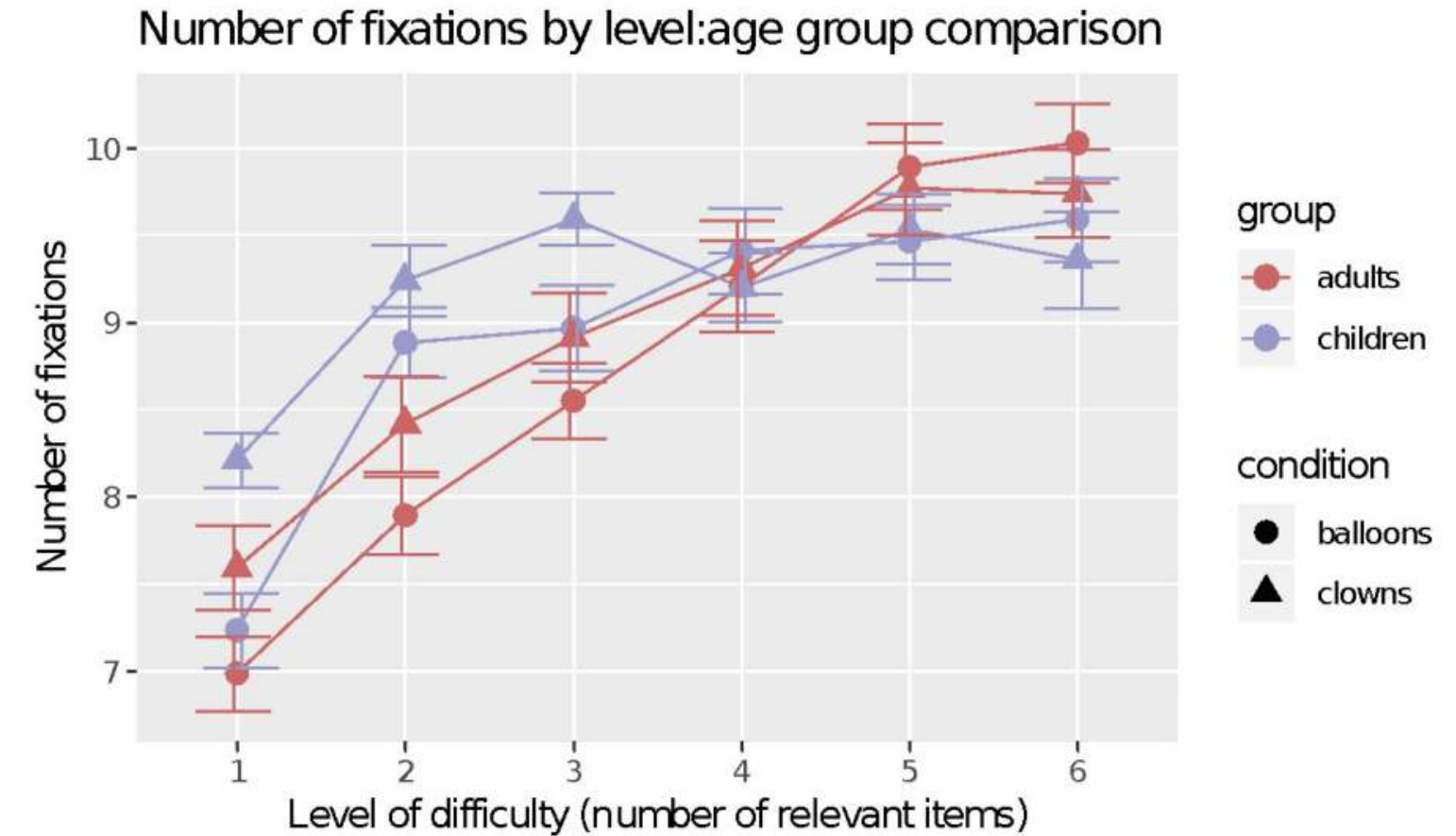

Figure 3. Fixations per difficulty level and age group Accuracy by level: age group comparison

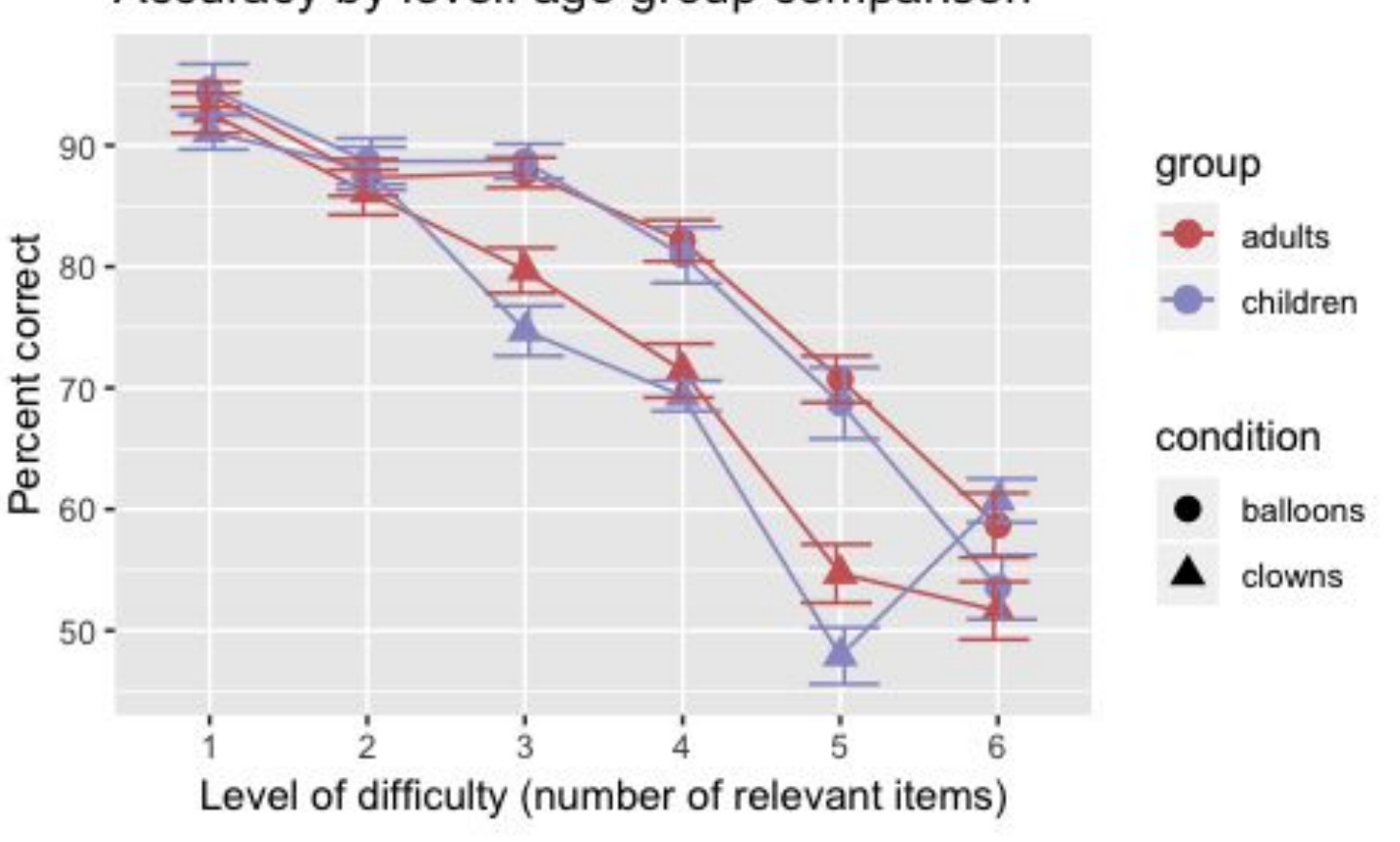

Figure 4. Accuracy per difficulty level and age group
Discussion

Number of fixations increase with difficulty as expected.

For children the number of fixations increased sharply after the first level of difficulty and then remained more stable.

According to predictions an effect of interference is found in terms of reaction time, and not according to prediction effect of interference was not found in terms of eye-movements indices. 